\title{
Optimal kinematic control of an Autonomous Underwater Vehicle
}

\author{
James Biggs and William Holderbaum
}

\begin{abstract}
This note investigates the motion control of an Autonomous Underwater Vehicle (AUV). The AUV is modeled as a nonholonomic system as any lateral motion of a conventional, slender AUV is quickly damped out. The problem is formulated as an optimal kinematic control problem on the Euclidean Group of Motions $S E(3)$, where the cost function to be minimized is equal to the integral of a quadratic function of the velocity components. An application of the Maximum Principle to this optimal control problem yields the appropriate Hamiltonian and the corresponding vector fields give the necessary conditions for optimality. For a special case of the cost function the necessary conditions for optimality can be characterized more easily and we proceed to investigate its solutions. Finally, it is shown that a particular set of optimal motions trace helical paths. Throughout this note we highlight a particular case where the quadratic cost function is weighted in such a way that it equates to the Lagrangian (Kinetic Energy) of the AUV. For this case the regular extremal curves are constrained to equate to the AUV's components of momentum and the resulting vector fields are the d'Alembert-Lagrange equations in Hamiltonian form.
\end{abstract}

Index Terms- Optimal Control, Underwater Vehicle, Nonholonomic systems.

\section{INTRODUCTION}

The motion control and path planning of Autonomous Underwater Vehicles (AUVs) has attracted a large amount of interest in recent years, see for example [1], [2], [3], [4], [5]. In this note we consider the optimal kinematic control of an AUV which is free to rotate but which is constrained to translate along a particular body axis (surge direction). This assumption is plausible for a conventional, slender AUV as any lateral motion (sway and heave directions) is quickly damped out.

Firstly, we relate the AUV's components of velocity to the curvature and torsion of the path that the AUV traces. This relation is derived by equating the kinematic description of the AUV to the evolution of a Serret-Frenet frame along the AUV's (non-degenerate) path. Following this a fixed end point optimal control problem is posed for the kinematic system with the velocities as the control inputs, where the cost function to be minimized is a quadratic function of the velocity components. An application of the Maximum Principle (see [6], [7]) to this optimal control problem then yields the appropriate Hamiltonian. Using the Poisson bracket we obtain the corresponding Hamiltonian vector fields which yield the necessary conditions for optimality. A particular case of the cost function is studied, that is, where two of its weights are equal. In this case the corresponding Hamiltonian vector fields can be solved analytically. Finally, we further specialize to optimal paths with constant surge rate and show

\footnotetext{
J. Biggs is with the Department of Mechanical Engineering, University of Strathclyde, Glasgow, United Kingdom james.biggsestrath.ac.uk

W. Holderbaum is with School of Systems Engineering, University of Reading, Reading, United Kingdom w.holderbaumerdg.ac.uk
}

that they correspond to helical paths. This approach illustrates an alternative method over the direct variational arguments in [8], [9], [10] and [11] used to treat these type of control problems.

Throughout this note we highlight a particular case of this optimal control problem, where the cost function is weighted in such a way that it equates to the Lagrangian (Kinetic Energy) of the AUV. If the regular extremal curves are constrained to equal the components of momentum of the AUV, the Poisson bracket no longer satisfies the Jacobi identity [9]. In this case the resulting vector fields are equivalent to the d'Alembert-Lagrange equations in Hamiltonian form (see for example [12]) for the AUV .

In the following section the kinematic equations of motion and the geometry of the AUV are stated and discussed.

\section{Kinematics AND GeOMETRY OF THE AUV}

The configuration space of the AUV travelling in Euclidean space $\mathbb{R}^{3}$, is represented by curves in the Special Euclidean Group $S E(3)$ :

$$
g(t)=\left(\begin{array}{ll}
1 & 0 \\
\gamma & R
\end{array}\right)
$$

where $\gamma \in \mathbb{R}^{3}$ describes the path that the AUV traces and $R \in S O(3)$ describes the orientation of the AUV at $\gamma$. The AUV travels at arbitrary speed $v=\frac{d \gamma}{d t}$ constrained to travel in the surge direction (lateral motions are damped out quickly due to viscous friction). The kinematics of the AUV are then described by

$$
\frac{d g(t)}{d t}=g(t)\left(v B_{1}+\Omega_{r_{1}} A_{1}+\Omega_{r_{2}} A_{2}+\Omega_{r_{3}} A_{3}\right)
$$

where $g(t) \in S E(3)$ with $v$ and $\Omega_{r_{i}}$ the linear and angular velocities respectively and where $A_{1}, A_{2}, A_{3}, B_{1}, B_{2}, B_{3}$ form a basis for the Lie algebra of $S E(3)$. The Lie bracket is defined as $[X, Y]=X Y-Y X$ for $X, Y \in \mathfrak{s e}(3)$ with the Lie algebra described by the following Lie bracket table:

\begin{tabular}{|c|c|c|c|c|c|c|}
\hline$[]$, & $A_{1}$ & $A_{2}$ & $A_{3}$ & $B_{1}$ & $B_{2}$ & $B_{3}$ \\
\hline$A_{1}$ & 0 & $A_{3}$ & $-A_{2}$ & 0 & $B_{3}$ & $-B_{2}$ \\
$A_{2}$ & $-A_{3}$ & 0 & $A_{1}$ & $-B_{3}$ & 0 & $B_{1}$ \\
$A_{3}$ & $A_{2}$ & $-A_{1}$ & 0 & $B_{2}$ & $-B_{1}$ & 0 \\
$B_{1}$ & 0 & $B_{3}$ & $-B_{2}$ & 0 & 0 & 0 \\
$B_{2}$ & $-B_{3}$ & 0 & $B_{1}$ & 0 & 0 & 0 \\
$B_{3}$ & $B_{2}$ & $-B_{1}$ & 0 & 0 & 0 & 0 \\
\hline
\end{tabular}

physically $A_{1}, A_{2}, A_{3}, B_{1}, B_{2}, B_{3}$ describe the infinitesimal motion of the AUV in the yaw, pitch, roll, surge, sway and heave directions respectively.

The path that this AUV traces $\gamma \in \mathbb{R}^{3}$ can be given a geometric interpretation (assuming that $\gamma$ is regular and free of points of inflection) by associating the kinematic equations 
(2) with a Serret-Frenet frame described by the differential equations:

$$
\begin{aligned}
& \frac{d \mathbf{T}}{d t}=\kappa v \mathbf{N} \\
& \frac{d \mathbf{N}}{d t}=-\kappa v \mathbf{T}+\tau v \mathbf{B} \\
& \frac{d \mathbf{B}}{d t}=-\tau v \mathbf{N}
\end{aligned}
$$

such that $\frac{d \gamma}{d t}=v \mathbf{T}$ where $\mathbf{T}$ is the tangent vector to the curve $\gamma, \mathbf{N}$ the normal vector and $\mathbf{B}$ the binormal vector with $\kappa$ and $\tau$ the curvature and torsion functions respectively.

The Serret-Frenet frame (3) is related to the kinematic equations (2) using the following theorem:

Theorem 1: The curvature $\kappa$ and torsion $\tau$ of the AUV's path is exclusively expressed in terms of its velocity components $v \neq 0, \Omega_{r_{1}}, \Omega_{r_{2}}, \Omega_{r_{3}} \neq 0$ :

$$
\begin{aligned}
& \kappa=\frac{\Omega_{r_{3}} \cos \beta-\Omega_{r_{2}} \sin \beta}{v} \\
& \tau=\frac{\frac{d \beta}{d t}+\Omega_{r_{1}}}{v}
\end{aligned}
$$

where $\beta$ is

$$
\tan \beta=-\frac{\Omega_{r_{2}}}{\Omega_{r_{3}}}
$$

Proof. The proof of this follows from [13] and the generalization to arbitrary speed $v$ is trivial. These equations will be used later in the note to deduce the optimal paths of the AUV.

\section{Optimal Kinematic CONTROL}

The motion control problem in this paper aims to derive optimal velocity inputs with respect to minimizing the integral of a quadratic cost function in the velocity components. Our motion control problem is formalized in the following Problem Statement:

Problem Statement 1: Compute the optimal kinematic controls and the corresponding curvature and torsion of the AUV's path, where the evolution of the AUV's configuration space $g(t) \in S E(3)$ is a solution of the left-invariant differential system (2) subject to minimizing the expression:

$$
J=\frac{1}{2} \int_{0}^{T} m_{1} v^{2}+c_{1} \Omega_{r_{1}}^{2}+c_{2} \Omega_{r_{2}}^{2}+c_{3} \Omega_{r_{3}}^{2} d t
$$

with the given boundary conditions $g(0)=g_{0}$ and $g(T)=g_{T}$, where $m_{1}, c_{1}, c_{2}, c_{3}$ are constant weights.

We note that if the weights $m_{1}, c_{1}, c_{2}, c_{3}$ are constrained in such a way that they equate to the components of mass $m_{1}$ and inertia $c_{1}, c_{2}, c_{3}$ then the cost function is analogous to the integral of the Lagrangian (Kinetic Energy) of the system. The tool used to tackle Problem Statement 1 is the coordinate free Maximum Principle of optimal control, see [6] and [7] for technical details. The Maximum Principle of optimal control identifies the appropriate left-invariant Hamiltonian $H$ on the dual of the Lie algebra $\mathfrak{s e}(3)^{*}$. The Hamiltonian corresponding to (2) and (6) is written as (see for example [6], [9]):

$H(p, u)=v p\left(g(t) B_{1}\right)+\sum_{i=1}^{3} \Omega_{r_{i}} p\left(g(t) A_{i}\right)-p_{0}\left(\frac{1}{2}\left(m_{1} v^{2}+\sum_{i=1}^{3} c_{i} \Omega_{r_{i}}^{2}\right)\right)$ where $p(\cdot): T_{g(t)} S E(3) \mapsto \mathbb{R}$ such that $p\left(g(t) B_{i}\right), p\left(g(t) A_{i}\right)$ are scalar components of an element in $T_{g(t)}^{*} S E(3)$, where $p_{0} \geqslant 0$ is a fixed positive constant. Because of the non-holonomic nature of this problem, the extremal curves that correspond to an optimal trajectory can be either abnormal or normal i.e. there are two types of Hamiltonian to consider. $p_{0}$ is set to 1 for regular extremals and 0 for abnormal extremals. In this paper we consider only regular extremals and set $p_{0}=1$. Following from the Maximum Principle and the fact that (7) is a concave function in $v, \Omega_{r_{i}}$ the optimal velocity inputs are given by $\frac{d H}{d v}=$ 0 and $\frac{d H}{d \Omega_{r_{i}}}=0$ it follows that:

$$
\begin{aligned}
& v=\frac{1}{m_{1}} p\left(g(t) B_{1}\right) \quad \Omega_{r_{1}}=\frac{1}{c_{1}} p\left(g(t) A_{1}\right) \\
& \Omega_{r_{2}}=\frac{1}{c_{2}} p\left(g(t) A_{2}\right) \quad \Omega_{r_{3}}=\frac{1}{c_{3}} p\left(g(t) A_{3}\right)
\end{aligned}
$$

As the configuration of the AUV is the Lie group $S E(3)$, the cotangent bundle $T^{*} S E(3)$ can be realized as the direct product $S E(3) \times \mathfrak{s e}(3)^{*}$ where $\mathfrak{s e}(3)^{*}$ is the dual of the Lie algebra [6]. Therefore, the original Hamiltonian defined on $T^{*} S E(3)$ can be expressed as a reduced Hamiltonian on the dual of the Lie algebra $\mathfrak{s e}(3)^{*}$. We define the linear functions $M_{i}=$ $p\left(g(t) A_{i}\right)=\hat{p}\left(A_{i}\right)$ and $p_{i}=p\left(g(t) B_{i}\right)=\hat{p}\left(B_{i}\right)$ for $i=1,2,3$, see [6]. Therefore, from (8) it follows that the maximizing inputs are:

$$
\begin{aligned}
& v=\frac{1}{m_{1}} p_{1}, \quad \Omega_{r_{1}}=\frac{1}{c_{1}} M_{1} \\
& \Omega_{r_{2}}=\frac{1}{c_{2}} M_{2}, \quad \Omega_{r_{3}}=\frac{1}{c_{3}} M_{3}
\end{aligned}
$$

In the particular case that the weights $c_{1}, c_{2}, c_{3}$ and $m_{1}$ equate to the components of inertia and mass respectively, the regular extremals $M_{1}, M_{2}, M_{3}$ and $p_{1}$ in (9) equate to the components of angular and linear momentum respectively. Substituting (9) into (7) gives the optimal Hamiltonian

$$
H=\frac{1}{2}\left(\frac{p_{1}^{2}}{m_{1}}+\frac{M_{1}^{2}}{c_{1}}+\frac{M_{2}^{2}}{c_{2}}+\frac{M_{3}^{2}}{c_{3}}\right)
$$

The function (10) is the appropriate Hamiltonian function for the given optimal control problem and for the particular case that $M_{1}, M_{2}, M_{3}, p_{1}$ are the components of momentum, the function (10) is the energy Hamiltonian of the AUV. The necessary conditions for optimality are then computed by making use of the Poisson bracket defined in terms of the Lie bracket $\{\hat{p}(\cdot), \hat{p}(\cdot)\}=-\hat{p}([\cdot, \cdot])$ which yields:

$$
\left\{\begin{array}{l}
\frac{d M_{1}}{d t}=\left\{M_{1}, H\right\}=\frac{-M_{2} M_{3}}{c_{2}}+\frac{M_{2} M_{3}}{c_{3}} \\
\frac{d M_{2}}{d t}=\frac{M_{1} M_{3}}{c_{1}}-\frac{M_{1} M_{3}}{c_{3}}+\frac{p_{1} p_{3}}{m_{1}} \\
\frac{d M_{3}}{d t}=\frac{-M_{1} M_{2}}{c_{1}}+\frac{M_{1} M_{2}}{c_{2}}-\frac{p_{1} p_{2}}{m_{1}} \\
\frac{d p_{1}}{d t}=\frac{-M_{2} p_{3}}{c_{2}}+\frac{p_{2} M_{3}}{c_{3}} \\
\frac{d p_{2}}{d t}=\frac{M_{1} p_{3}}{c_{1}}-\frac{p_{1} M_{3}}{c_{3}} \\
\frac{d p_{3}}{d t}=-\frac{M_{1} p_{2}}{c_{1}}+\frac{p_{1} M_{2}}{c_{2}}
\end{array}\right.
$$


where $M_{1}, M_{2}, M_{3}, p_{1}, p_{2}, p_{3} \in \mathfrak{s e}^{*}(3)$ are the extremal curves. In the particular case that $M_{1}, M_{2}, M_{3}$ and $p_{1}$ equate to the components of momentum through equation (9) and further constraining the extremals $p_{2}$ and $p_{3}$ to equal the components of momentum, then $p_{2}$ and $p_{3}$ are zero (as the components of velocity are zero in these directions) and we have $\left\{p_{2}, F\right\}=0$ and $\left\{p_{3}, F\right\}=0$ for any function $F \in \mathfrak{s e}^{*}(3)$. In this case the Poisson bracket does not satisfy the Jacobi identity and we have an almost-Poisson bracket [9]. The Hamiltonian vector fields computed using the almost-Poisson bracket are:

$$
\left\{\begin{array}{l}
\frac{d M_{1}}{d t}=\frac{-M_{2} M_{3}}{c_{2}}+\frac{M_{2} M_{3}}{c_{3}} \\
\frac{d M_{2}}{d t}=\frac{M_{1} M_{3}}{c_{1}}-\frac{M_{1} M_{3}}{c_{3}} \\
\frac{d M_{3}}{d t}=\frac{-M_{1} M_{2}}{c_{1}}+\frac{M_{1} M_{2}}{c_{2}} \\
\frac{d p_{1}}{d t}=0
\end{array}\right.
$$

where $M_{1}, M_{2}, M_{3}, p_{1}$ are the AUV's components of momentum. In this case the optimal control problem has been constrained in such a way that the vector fields (12) are equivalent to the d'Alembert-Lagrange equations in Hamiltonian form. This illustrates that the d'Alembert-Lagrange equations of the AUV, are a special constrained case of the general optimal control problem. We proceed to investigate the general necessary conditions for optimality (11). In the next section we observe a case of the cost function (6) where the extremal curves defined by (11) can be characterized more easily and we proceed to investigate them.

\section{A SPECIAL CASE OF THE COST FUNCTION}

In this section we investigate a special case of the cost function (6) where the weights $c_{2}$ and $c_{3}$ are equal. For simplicity of exposition we normalize the constants such that $c_{2}=c_{3}=1$. This condition gives $\frac{d M_{1}}{d t}=0$ in (11) and therefore $M_{1}$ is constant (denote $\sigma$ ). In addition to the constant $M_{1}=\sigma$ we recall the Casimir functions that exist for any left-invariant Hamiltonian system on $S E(3)$ (see [6]):

$$
\begin{gathered}
I_{2}=p_{1}^{2}+p_{2}^{2}+p_{3}^{2} \\
I_{3}=p_{1} M_{1}+p_{2} M_{2}+p_{3} M_{3}
\end{gathered}
$$

where $I_{2}$ and $I_{3}$ are constant along the Hamiltonian flow: $\left\{H, I_{2}\right\}=0,\left\{H, I_{3}\right\}=0$ and $\left\{I_{2}, I_{3}\right\}=0 . \sigma, H, I_{2}, I_{3}$ provide four integrals of motion which is sufficient to reduce the 6-dimensional non-canonical Hamiltonian system to a completely integrable 2 dimensional Hamiltonian system, as in [5]. We proceed to solve for the extremal control inputs (9), firstly from (11) we have:

$$
\frac{d p_{1}}{d t}=p_{2} M_{3}-M_{2} p_{3}
$$

it follows that

$$
\left(\frac{d p_{1}}{d t}\right)^{2}=p_{2}^{2} M_{3}^{2}+p_{3}^{2} M_{2}^{2}-2 p_{2} p_{3} M_{2} M_{3}
$$

Using (10) and (13) write;

$$
\begin{aligned}
& 2 H-\frac{p_{1}^{2}}{m_{1}}-\frac{\sigma^{2}}{c_{1}}=M_{2}^{2}+M_{3}^{2} \\
& I_{2}-p_{1}^{2}=p_{2}^{2}+p_{3}^{2}
\end{aligned}
$$

multiplying the two equations in (17) gives:

$$
\begin{aligned}
& \left(I_{2}-p_{1}^{2}\right)\left(2 H-\frac{p_{1}^{2}}{m_{1}}-\frac{\sigma^{2}}{c_{1}}\right)= \\
& p_{2}^{2} M_{2}^{2}+p_{2}^{2} M_{3}^{2}+p_{3}^{2} M_{2}^{2}+p_{3}^{2} M_{3}^{2}
\end{aligned}
$$

To find explicit solutions it is necessary to use the Casimir function (14) and rearranging write:

$$
I_{3}-p_{1} \sigma=p_{2} M_{2}+p_{3} M_{3}
$$

squaring (19) yields:

$$
\left(I_{3}-p_{1} \sigma\right)^{2}=p_{2}^{2} M_{2}^{2}+p_{3}^{2} M_{3}^{2}+2 p_{2} M_{2} p_{3} M_{3}
$$

finally substituting (20) and (18) into (16) and simplifying gives the following quartic function:

$$
\begin{aligned}
& \left(\dot{p}_{1}\right)^{2}=\frac{p_{1}^{4}}{m_{1}}+\left(\frac{\sigma^{2}}{c_{1}}-\sigma^{2}-\frac{I_{2}}{m_{1}}-2 H\right) p_{1}^{2} \\
& +\left(2 I_{3} \sigma\right) p_{1}+\left(2 H I_{2}-I_{3}^{2}-\frac{I_{2} \sigma^{2}}{c_{1}}\right)
\end{aligned}
$$

The function (21) is a quartic function of $p_{1}$ and can therefore be solved by an elliptic function, see [14]. $p_{1}$ can be expressed as a linear function of a Weierstrass' $\wp$-function [14], however, this is a complex symbolic expression and is omitted from this note. The remaining extremal curves will be expressed in terms of the analytic function $p_{1}$. To solve for the remaining extremal curves $M_{2}$ and $M_{3}$ the Hamiltonian function (10) is used. Recall that $M_{1}$ is a constant $\sigma$ and therefore the reduced Hamiltonian satisfies

$$
M_{2}^{2}+M_{3}^{2}=2 H-\frac{p_{1}^{2}}{m_{1}}-\frac{\sigma^{2}}{c_{1}}
$$

This suggests using polar coordinates for $M_{2}$ and $M_{3}$ :

$$
M_{2}=r \sin \theta, \quad M_{3}=r \cos \theta
$$

$r$ is given by substituting (23) into (22):

$$
r=\left(2 H-\frac{p_{1}^{2}}{m_{1}}-\frac{\sigma^{2}}{c_{1}}\right)^{1 / 2}
$$

and $\theta$ is given as follows:

$$
\begin{gathered}
\theta=\arctan \left(\frac{M_{2}}{M_{3}}\right) \\
\dot{\theta}=\frac{M_{3} \dot{M}_{2}-M_{2} \dot{M}_{3}}{M_{2}^{2}+M_{3}^{2}}
\end{gathered}
$$

substituting in the values for $\dot{M}_{2}$ and $\dot{M}_{3}$ from (11) and simplifying gives:

$$
\dot{\theta}=\frac{\sigma}{c_{1}}-\sigma+\frac{p_{1}\left(p_{2} M_{2}+p_{3} M_{3}\right)}{m_{1}\left(M_{2}^{2}+M_{3}^{2}\right)}
$$

then substituting in the equations (14) and (10) into (27) yields:

$$
\dot{\theta}=\frac{\sigma}{c_{1}}-\sigma+\frac{p_{1}\left(I_{3}-p_{1} \sigma\right)}{m_{1}\left(2 H-\frac{\sigma^{2}}{c_{1}}-\frac{p_{1}^{2}}{\sigma}\right)}
$$


generally equation (28) can be solved numerically and in some special cases analytically (an example is shown later). From the components of velocity we can also compute the curvature and torsion of the AUV's path using the equations in (4). Recall that with $c_{2}=c_{3}=1$ the optimal inputs (9) are of the form:

$$
\begin{aligned}
& v=\frac{p_{1}}{m_{1}} \quad \Omega_{r_{1}}=\frac{M_{1}}{c_{1}}=\frac{\sigma}{c_{1}} \\
& \Omega_{r_{2}}=M_{2}=r \sin \theta \quad \Omega_{r_{3}}=M_{3}=r \cos \theta
\end{aligned}
$$

to compute the optimal curvature and torsion of the AUV's path, substitute the optimal inputs (29) into (4) and simplify to get:

$$
\kappa=\frac{m_{1} r}{p_{1}}, \quad \tau=\frac{m_{1}\left(\sigma / c_{1}-\dot{\theta}\right)}{p_{1}}
$$

with $p_{1} \neq 0$ and therefore the curvature and torsion of the AUV's path are determined completely in terms of constants and the extremal curve $p_{1}$.

\section{Optimal Helical Motions}

In this section a particular set of optimal motions are investigated, that is, optimal motions that are shown to trace helical paths. These particular motions correspond to equilibrium points of equation (21), that is where $\dot{p}_{1}=0$.

The phase portrait of equation (21) given in Figure 1 represents contours for different values of $H$. The equilibrium points are given where the trajectory intersects the $\mathrm{x}$-axis $\left(\dot{p}_{1}=0\right)$.

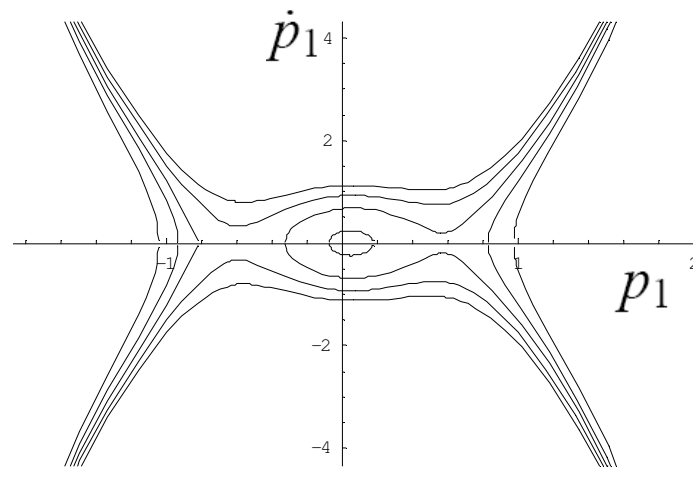

Fig. 1. Phase portrait of the extremal curve $p_{1}$

At $\dot{p}_{1}=0$ the functions $p_{1}, r, \dot{\theta}$ defined by equations $(21,24,28)$ are constant and therefore from (30) it follows that $\kappa$ and $\tau$ are constant, which in turn implies that helices are optimal paths for weights of the cost function $c_{2}=c_{3}=1$. In addition we observe that the solution (29) at $\dot{p}_{1}=0$ is also a solution of the dynamic equations of motion (12) when $c_{2}=c_{3}$. Therefore, these particular optimal motions are also solutions of the dynamic equations of motion of a laterally constrained axisymmetric AUV. This particular set of optimal motions make available useful and simplistic reference paths for a real AUV to track. Moreover, the particular optimal helical motions could provide tracking trajectories for ascending and descending conventional, slender AUVs.

\section{CONCLUSION}

This note has investigated the motion control of an Autonomous Underwater Vehicle (AUV). The AUV is treated as a nonholonomic system as any lateral motion of a conventional, slender AUV is quickly damped out. The problem is formulated as a fixed end point optimal control problem on the Euclidean Group of Motions $S E(3)$, where the cost function to be minimized is equal to the integral of a quadratic function of the velocity components. An application of the Maximum Principle to this optimal control problem yields the appropriate Hamiltonian and along with the Poisson bracket we derive the Hamiltonian vector fields, which define the necessary conditions for optimality. For the special case of the cost function (two of the weights are equal) the necessary condition for optimality can be characterized more easily and we proceed to investigate these solutions. Finally, it is shown that a particular set of optimal motions trace helical paths. Throughout the note we highlight a particular case where the quadratic cost function is weighted in such a way that it equates to the Lagrangian (Kinetic Energy) of the AUV. Further constraining the extremal curves to equate to the components of momentum defines an almost-Poisson bracket and the resulting vector fields are equivalent to the d'AlembertLagrange equations in Hamiltonian form.

\section{REFERENCES}

[1] Woolsey, C., Leonard, N.,'Stabilizing Underwater Vehicle Motion Using Internal Rotors'. Automatica, Vo. 38, No. 12, pp. 2053-2062.

[2] Leonard, N. E., Graver, J. G.,'Model-Based Feedback Control of Autonomous Underwater Vehicles' IEEE Journal of Oceanic Engineering: Special Issue on Autonomous Ocean sampling Networks, pp. 633-645, 2001.

[3] Schultz, C., Woolsey, C., 'An Experimental Platform for Validating internal actuator control strategies' Proceedings of the IFAC workshop on Guidance and Control of Underwater Vehicles, pp.209-214, 2003.

[4] Leonard, N., Krishnaprasad, P. S., 'Motion control of drift free, leftinvariant systems on Lie groups'. IEEE Transactions on Automatic control, vol. 40, pp.1539-1554, 1995.

[5] Holmes, P., Jenkins, J., Leonard, N.E., 'Dynamics of the Kirchhoff equations: Coincident centers of gravity and buoyancy'. Physica D 118 , p. 311-342, 1998.

[6] Jurdjevic, V., 'Geometric Control Theory'. Advanced Studies in Mathematics, Cambridge University Press, 52,1997.

[7] Sussmann, H. J., 'An introduction to the coordinate-free maximum principle'. In Geometry of Feedback and Optimal Control,B. Jakubczyk and W. Respondek Eds., Marcel Dekker, New York, pp. 463-557, 1997.

[8] Crouch, P., Silva Leite, F., 'The Dynamic interpolation problem: On Riemannian manifolds, Lie groups and Symmetric spaces'. Journal of Dynamical and control systems, Vol.1, No. 2, p177-202, 1995.

[9] Bloch, A. M., 'Nonholonomic Mechanics and Control'. Springer-Verlag, New York,2003.

[10] Bloch, A., Crouch, P., Ratui, T., 'Sub-Riemannian optimal control problems'. Fields Institute Communications, vol. 3, pp. 35-48, 1994.

[11] Hussein, I. I., Bloch, A. M.,'Constrained Optimal Trajectory Tracking on the Group of Rigid Body Motions'. Proceedings of IEEE CDC-ECC, Seville, pp. 2152- 2157, 2005.

[12] van der Schaft, A. J, Maschke, B. M., 'On the Hamiltonian formulation of nonholonomic mechanical systems'. Reports on Mathematical Physics, vol. 34, 1994.

[13] Biggs, J. D., Holderbaum, W., Jurdjevic, V., 'Singularities of Optimal Control Problems on some 6-D Lie groups'. IEEE Transactions on Automatic Control, pp. 1027-1038, June 2007.

[14] Lawden, D., 'Elliptic functions and applications'. Springer-Verlag, New York, 1989. 